\title{
An unusual case of diaphragmatic hernia
}

\author{
Banagala ASK, Shrikharan S, Nanayakkara KAMKK, Ruwanpathirana HSK, Liyanage AS \\ Colombo South Teaching Hospital, Kalubowila, Dehiwala, Sri Lanka
}

Correspondence: Dr.A.S.K. Banagala;

Mobile:+94714166548; e-mail: anurabsurg@gmail.com

\section{Case report}

A 27-year-old man was admitted to the medical casualty with severe left sided chest pain and repeated vomiting. On admission, he was acutely ill and restless. Radial pulse was of low volume and fast at $100 / \mathrm{min}$ while blood pressure was $80 / 60 \mathrm{mmHg}$. The trachea had shifted to the right side and air entry to the left chest was markedly reduced. The opacity seen in the chest radiograph was initially considered to be a large left sided pleural effusion. A chest drain was inserted into the left pleural cavity, but there was only a little improvement of the patient's clinical picture (Figure 1). The patient was reviewed and the diagnosis of obstructed left diaphragmatic hernia was made. Abdominal radiographs were not of much help.

After resuscitation, a nasogastric tube was passed and the patient was given general anaesthesia. A midline upper abdominal incision was made and extended through the seventh intercostal space into the thorax due to the difficulty of delivering the contents of the hernial sac which included the spleen, left adrenal gland, most of the transverse colon, splenic flexure of the colon and the body of the stomach (Figure $2 \mathrm{~A} \& \mathrm{~B}$ ). The defect was found to be located posterolaterally in the peripheral part of the left hemidiaphragm and the peripheral muscular part of the diaphragm had to be divided to reduce the colon, spleen and the stomach back into the peritoneal cavity (Figure 3).

\section{Discussion}

Late presentation of congenital diaphragmatic hernia is reported to be $5-25 \%$ (1). Acute respiratory distress seems to be a more common presentation than acute intestinal obstruction. Causes of late presentation are either due to late rupture of the hernial sac that contained the viscera or plugging of the hernial defect by solid organs such as the spleen prior to herniation of hollow viscera into the chest (2). The usual left sided hernia contain small bowel, spleen, stomach and colon whereas right sided herniae may contain liver and intestines (2). Small defects tend to present with obstruction / strangulation of viscera whilst large defects present with respiratory distress and compromised circulation (3). Misinterpretation of the chest radiograph as a pleural effusion is common in this condition and leads to insertion of chest drains and delay in life saving surgery as seen in our case (4). Obtaining a chest radiograph after insertion of a nasogastric tube is useful in arriving at a correct diagnosis.

The mass effect of the intrathoracic viscera causes mediastinal shift, kinks the vena cava and pulmonary veins resulting in reduced cardiac output. Emergency surgery is needed to reduce the contents of the hernia and to repair the diaphragmatic defect. Most hernias could be reduced abdominally but rarely thoracotomy is necessary. Non-absorbable suture is used to repair the defect together with chest drainage and manual inflation of the collapsed lung prior to closure of the chest.

Important anaesthetic considerations are fast fluid resuscitation via large bore intravenous access, passage of nasogastric tube and aspiration of gastric contents, antacid premedication, and rapid sequence induction with cricoid pressure to prevent / minimise aspiration pneumonitis. Difficult intubation may necessitate awake fibreoptic intubation or tracheostomy under local anaesthesia. Face mask ventilation and nitrous oxide should be avoided to prevent gastric insufflation and distension which would worsen thoracic mass effect / mediastinal shift. Re-expansion of the collapsed lung prior to thoracotomy too would exacerbate the mass effect. 
Therefore a double lumen tube should be used to ventilate the normal lung alone initially with small tidal volumes and low pressures. Alternatively, a single lumen tube with a bronchial blocker may be used (4).

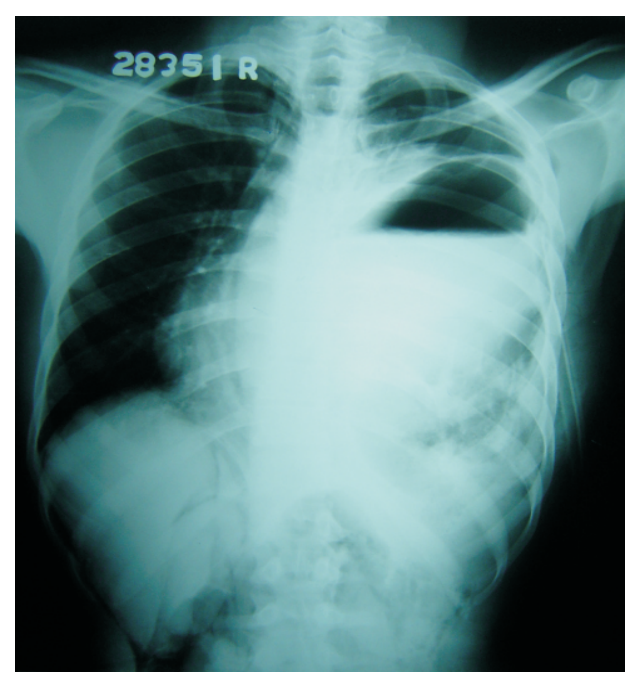

Figure 1: Erect Chest Radiograph after insertion of the intercostal tube
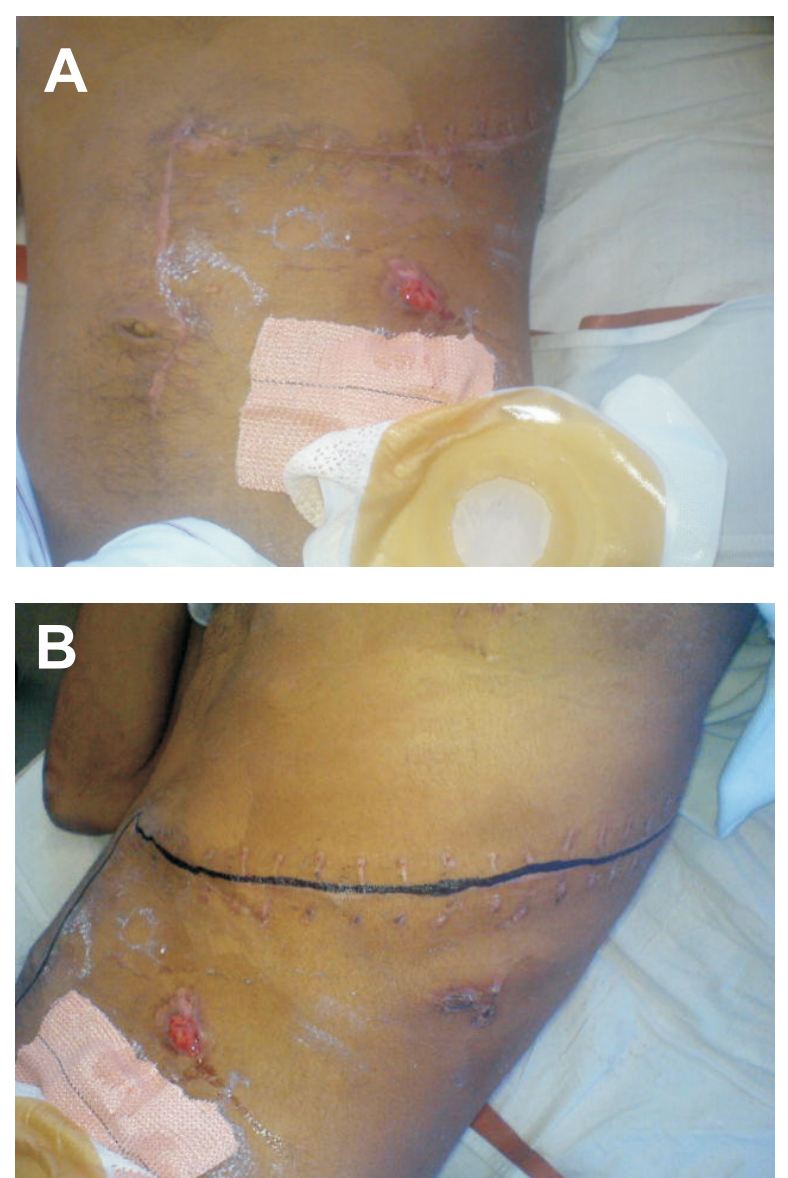

Figure 2: Healed thoraco-abdominal incision with the site of gastrostomy tube insertion

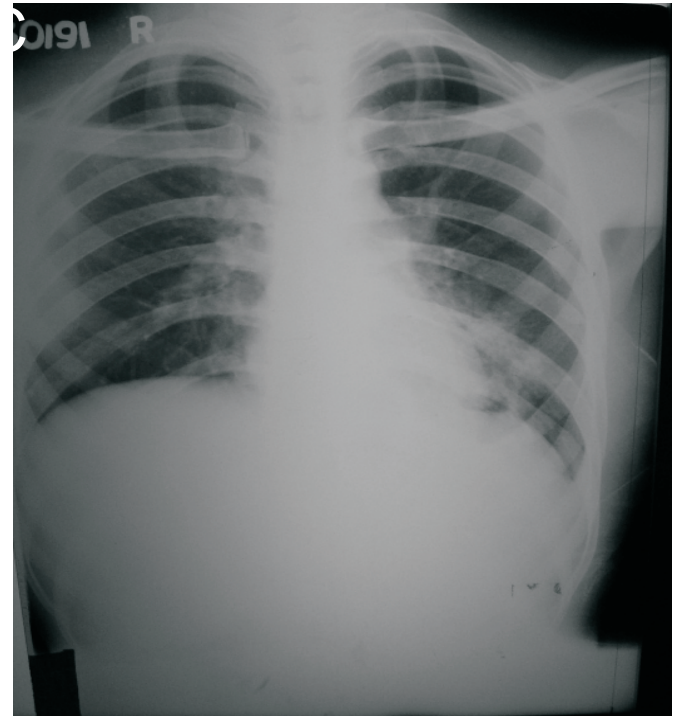

Figure 3: Postoperative chest radiograph after removal of the intercostal tube

\section{References}

1. Christiansen LA, Blichert-Toft M, Bertelsan S. Strangulated diaphragmatic hernia, a clinical study. Am J Surg 1975: 129: 574-8.

2. Rawat JD, Kureel SN, Tendon RK, Tendon S, Wakhar AK. Obstructed diaphragmatic hernia. J Indian Assoc Paed Surgeons 1999; 4: 34-8.

3. Bhardwaj M, Taxak S, Rattan KN, Goyal P, Aggrawal M. Late presentation of congenital diaphragmatic herniaAnaesthetic considerations The Internet Journal of Anesthesiology 2008; 16: 2.

4. Gupta S, Raiger LK, Shah D, Kumar S. Late presentation of congenital Bochdalek hernia. Indian Journal of Anaesthesia 2005 Dec: 499-503. 